\title{
Effective Acquisition of Dense Anisotropic BRDF
}

\author{
Jiří Filip ${ }^{1}$, Radomír Vávra ${ }^{1,2}$, Michal Havlíček ${ }^{1}$ \\ ${ }^{1}$ Institute of Information Theory and Automation of the ASCR, Czech Republic \\ ${ }^{2}$ Faculty of Information Technology, Czech Technical University in Prague \\ Email: filipj@utia.cas.cz
}

\begin{abstract}
The development of novel analytical BRDF models, as well as adaptive BRDF sampling approaches, rely on the appropriate BRDF measurement of real materials. The quality of measurements is even more critical when it comes to accurately representing anisotropic materials where the character of anisotropy is unknown (locations of anisotropic highlights, their width, shape, etc.). As currently there is a lack of dense yet noise-free BRDF anisotropic datasets, we introduce such unique measurements of three anisotropic fabric materials. In this paper we discuss a method of dense BRDF data acquisition, postprocessing, missing values interpolation, and analyze properties of the datasets. Our results are compared with photographs, dense data fitted and generated by two state-of-the art anisotropic BRDF models, and alternative measurements available.
\end{abstract}

\section{INTRODUCTION}

Representation of spatially homogeneous materials by BRDF was first outlined by Nicodemus et al. [1] and later widely adopted in computer graphics. A monospectral BRDF is a four-dimensional function $\operatorname{BRDF}\left(\theta_{i}, \theta_{v}, \varphi_{i}, \varphi_{v}\right)$ of illumination direction $\mathbf{I}=\left[\theta_{i}, \varphi_{i}\right]$ and view direction $\mathbf{V}=\left[\theta_{v}, \varphi_{v}\right]$. Because multispectral BRDF measurements are costly, spectral information is often approximated by means of three color channels viewed as a collection of three monospectral BRDFs. The four dimensions represent four mechanical degrees of freedom (DOF) realized when the BRDF is acquired. Although the number of DOF can be lowered using imagebased techniques, mirrors [2], or prior geometrical knowledge of measured sample [3], [4], all these approaches introduce noise and therefore some cannot be used for anisotropic measurements. Due to a complicated measurement process, many approaches resort to three-dimensional isotropic BRDF measurement only $\operatorname{BRDF}\left(\theta_{i}, \theta_{v}, \varphi_{i}-\varphi_{v}\right)$, i.e., reflectance is invariant to the rotation of a sample around its normal for fixed camera and light positions.

Our primary motivation was due to a lack of dense reference BRDF measurements suitable not only for the fitting of analytical models and their development, but especially for the development and evaluation of adaptive measurement techniques of anisotropic materials. Consequently, we use a highly precise gonioreflectometer-based setup for BRDF measurement. We assume that the size of measured material is comparable to its distance to light and camera. Due to varying illumination and viewing angles across the material's surface, a large variety of incoming and outgoing directions can be measured.

Contributions: Innovative is the introduction of accurate dense BRDF measurements of three anisotropic fabric materials, that are publicly available. We have shown the way a gonioreflectometer can be combined with image-based methododology to achieve reasonable measurement times while still

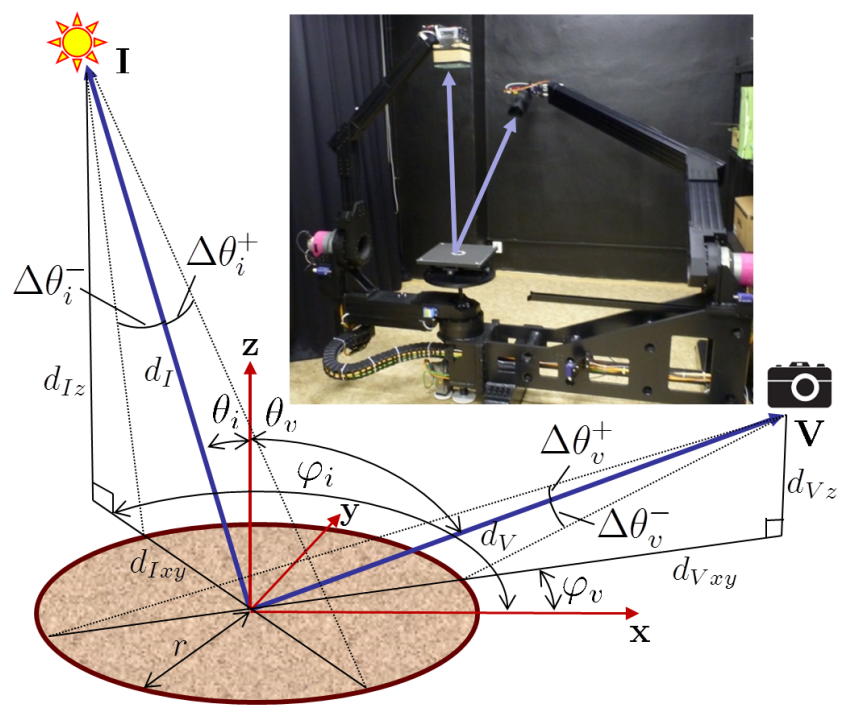

Fig. 1. The setup and scheme of BRDF measurement based on viewing and illumination angles variations across the measured surface.

yielding very accurate dense BRDF data. Through BRDF interpolation, we achieve uniform angular step $2^{\circ}$ in all four dimensions. To our best knowledge such dense data are not available yet.

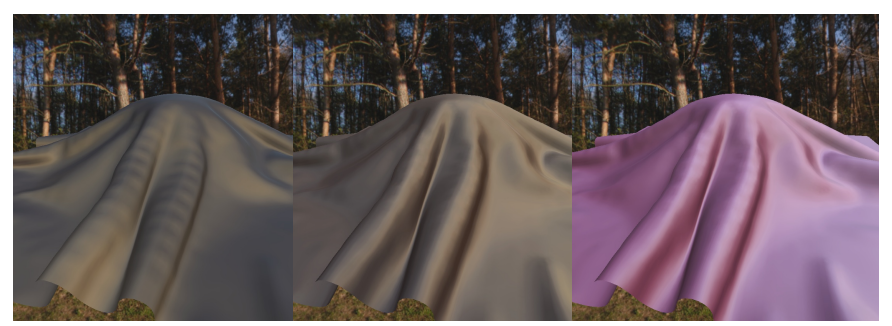

Fig. 2. Rendering of the measured dense BRDFs in environment illumination.

The paper is structured as follows. Section II sets the work in the context of previous research. Section III describes the data acquisition procedure, and Section IV explains further data processing and interpolation steps. Section V analyses properties of the measured material samples, while Section VI discusses the results and compares them with other approaches of dense BRDF data acquisition.

\section{PRIOR WORK}

Due to relative sparsity and noise presented in a typical BRDF measurement, material appearance representation applications resorted to simplified analytical BRDF models [5]. While their main advantage is the effective suppression of 
outliers and ability to provide data for arbitrary directions, their complicated fitting process often remains in a local minimum and some of the appearance features are often not captured properly. These problems are even more distinct for anisotropic materials requiring a more complicated model. To achieve better performance of these models or even avoid their need altogether, dense and accurate BRDF measurements are vital.

Although a vast body of research work deals with isotropic BRDF measurements only, we will briefly categorize the measurement approaches and then restrict ourselves to discussion of anisotropic BRDF measurement approaches.

In general, anisotropic BRDF can be obtained by gonioreflectometers sequentially sampling all four dimensions of BRDF [6], [7]. To speed up this process, mirror-based setups are used to thus reduce the number of mechanical parts. This is done by acquiring multiple views in a single image through means of projecting surface reflectance using hemispherical [8], [9], parabolic [10], [9], or ellipsoidal [11] mirrors. While, these solutions generally allow faster measurements, lower accuracy or a lower range of illumination/viewing angles often result. Lastly, image-based setups reduce mechanical complexity through the use of specifically shaped convex samples instead of mirrors. In [12] a sphere was used for isotropic BRDF measurements, while [13], [4] used a cylinder for anisotropic BRDF measurements.

In [11] an anisotropic BRDF measurement setup based on an ellipsoidal mirror and projector has been introduced. Although the setup allows measurement in a couple of minutes, it is limited by the low dynamic range of the projector and low overall accuracy of the setup, as it relies on accurate placement of the sample and uniform properties of the mirror.

The likely one of the first attempts of effective anisotropic BRDF measurement was presented in [14], where the number of mechanical elements was reduced by placing strips cut from measured material in different orientations on a cylinder of known geometry. This approach was further revived in a more recent work [4], where 20 strips of sample surface were attached to a cylinder. Light is moving around the rotating cylinder and a fixed camera captures its images. Its main limitation is a substantial measurement time (16 hours) and relatively noisy results.

To overcome these limitations, we propose to extend an idea of isotropic BRDF measurement [15], based on capturing variable incoming and outgoing BRDF directions in images of a flat sample size comparable to its distance to light and sensor. We used a gonioreflectometer setup and measured data were further processed and interpolated in four dimensions using uniform sampling step $2^{\circ}$ with maximal elevation $86^{\circ}$, i.e., to float array of dimension $\left|\theta_{i}\right| \times\left|\theta_{v}\right| \times\left|\varphi_{i}\right| \times\left|\varphi_{v}\right|=44 \times 44 \times$ $180 \times 180$. To our best knowledge, such dense yet still accurate anisotropic BRDF data are not available thus far; therefore, we believe our measurements will contribute to design and verification of BRDF models, study of real-material properties, or design of adaptive sampling methods [16].

\section{ACQUisition SETUP}

We used a gonioreflectometer setup (shown in Fig. 1) with high angular accuracy of light and sensor positioning $\left( \pm 0.03^{\circ}\right)$ as introduced in [16]. The image of the measured material sample was taken 8505 times. Where 6561 images represent uniform sampling of the hemisphere above the material surface using $81 \times 81$ viewing and illumination directions uniformly covering the hemisphere [17]. The remaining $1944=81 \times 24$ samples represent additional oblique illumination directions at elevation angle $\theta_{i}=85^{\circ}$ using azimuthal step $24^{\circ}$.

The measurement principle is based on the fact that an image of flat surface of size relative to its distance of light and camera captures a variety of illumination and viewing directions. This is due to varying elevation and azimuthal angles across the surface of measured material, as shown for elevation angles in Fig. 1. For elevations $\theta_{i}, \theta_{v}$ of the gonioreflectometer arms (valid in the image center only) can be computed the effective span of illumination and view elevation angles $\left(\Delta \theta^{-}, \Delta \theta^{+}\right)$in a single image as

$$
\begin{gathered}
d_{x y}=d \cdot \sin \theta \quad d_{z}=d \cdot \cos \theta \\
\Delta \theta^{+}=\operatorname{atan}\left(\frac{d_{x y}+r}{d_{z}}\right)-\theta \quad \Delta \theta^{-}=\operatorname{atan}\left(\frac{d_{x y}-r}{d_{z}}\right)-\theta
\end{gathered}
$$

A span of azimuthal angles $\varphi$ can be evaluated similarly. The effective span decreases as a function of actual view and illumination elevation angles as shown in Fig. 3. This span is higher for illumination elevation due to the fact that the physical distance of the light $(1.1 \mathrm{~m})$ to the measured sample (diameter $0.55 \mathrm{~m})$ is smaller than distance to the camera $(1.8$ $\mathrm{m})$.

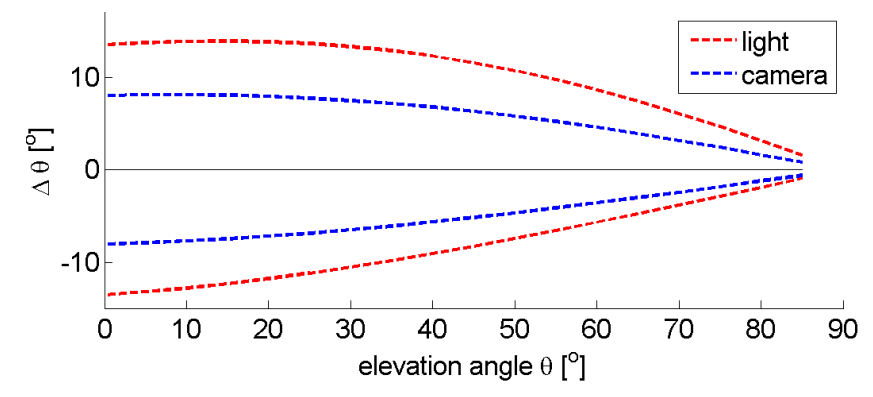

Fig. 3. Range of effective span of illumination and view elevation angles as a function of elevation angle $\left(\theta_{i}, \theta_{v}\right)$ in our measurement setup.

Our measurement target consists of a flat desk with registration marks and measured material as illustrated in Fig. 4. The measured fabric samples were flattened and stretched using pins. We used the Sobel filter for the marks edge detection and Hough transformation for lines detection. The lines intersections are used for homography computation and image rectification. The effective radius of the material is $r=277 \mathrm{~mm}$, and we sample it in rectified images using a uniform grid of samples $14 \mathrm{~mm}$ apart Fig. 4. All samples are bined to a matrix of dimension $\left|\theta_{i}\right| \times\left|\theta_{v}\right| \times\left|\varphi_{i}\right| \times\left|\varphi_{v}\right|=44 \times 44 \times 180 \times 180$, i.e., with angular step $2^{\circ}$. Sample values are taken as median value from neighborhood $14 \times 14 \mathrm{~mm}(50 \times 50$ pixels $)$ to suppress material geometry imperfections (folds, textile defects, etc.). This number of samples balances well their overall density to their visual contribution in the final data. The slight spatial non-homogenity is the main source of noise in the data; however, it can be effectively filtered by computing the median of samples present in each bin. 


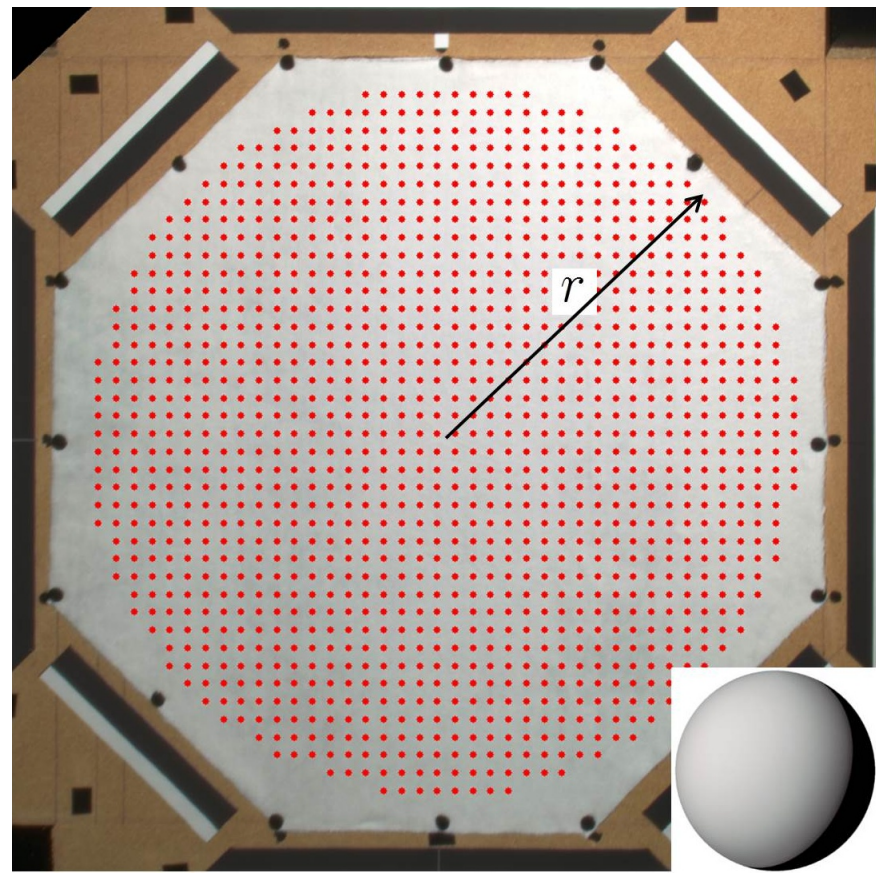

Fig. 4. Measured material sample of width $700 \mathrm{~mm}$, with uniform distribution of sampling points $14 \mathrm{~mm}$ apart within circle of radius $277 \mathrm{~mm}$. Bottom-right inset image shows rendering of the reference material used for light nonuniformity compensation.

\section{DATA PROCESSING AND COMPENSATION}

Camera occlusion removal - As our measurement setup consists of two arms, it is necessary to deal with material occlusion by arm holding the light. The occlusions are detected using Otsu's histogram-based binary segmentation algorithm for reference diffuse white fabric material (see Fig. 4). These precomputed occlusion masks are stored and used for removal of incorrect samples.

Illumination non-uniformity compensation - Further light attenuation across each image has to be compensated in each image individually. We used again the reference material (see Fig. 4). First, its sparse BRDF $(81 \times 81$ values $)$ is obtained by sampling only central points in each image; i.e., ideal values non-affected by the attenuation. This information is further interpolated using Radial basis functions (RBF) [18] to fill in a matrix of dense BRDF. Then during binning of the samples we take image value I, reference material value at the same position $I_{R}$, and interpolated central value corresponding to the same bin $I_{C}$. Final compensated value is obtained as

$$
I_{F}=I \cdot \frac{I_{C}}{I_{R}} \cdot \frac{1}{\cos \theta_{i}},
$$

where $\cos \theta_{i}$ compensates for illuminated area foreshortening. After the interpolation, the BRDF reciprocity was enforced by the averaging of reciprocal values.

Missing values interpolation - Binning angles over a spatial grid $14 \mathrm{~mm}$ apart, distributed uniformly in a circle of radius $r=277 \mathrm{~mm}$, provides 2810130 unique combinations of illumination and viewing angles in $2^{\circ}$ angular binning. As the BRDF resolution with angular step $2^{\circ}$ comprises almost 60 million values, it is necessary to interpolate missing values from measured samples. We used localized four-dimensional radial basis functions (RBF) interpolation [18]. For each of the unknown values, 200 surrounding values were found having the closest distance of illumination and view directions to the interpolated value. For a faster search of the closest samples we applied kd-tree data structure ${ }^{1}$. The RBF predicts the value based on these neighboring values and in comparison with other interpolation approaches (e.g., Kriging) provides reasonable speed.

Time frame - The measurement of 8505 HDR images of material surface takes about twenty hours. Collecting the data from the measured images and their compensation by the reference material adds another six hours, where the main overhead represents the loading of HDR images. The entire BRDF interpolation using the 200 closest values takes twelve hours using three cores of Intel Xeon E5-2643 3.3GHz.

\section{Measured Materials}

We measured three highly anisotropic material samples as shown in Fig. 5. Material fabricl12 consists of two types of threads (gold and dark-green), that produces distinct anisotropic behavior. The threads are interwoven in a plain pattern so that the proportion of threads visibility is about 40 vs. $60 \%$. The remaining two satin-like materials (fabric135 and fabric136), consist of a combination of diffuse dark-brown/red thread and tiny transparent nylon fibers covering almost $40 \%$ of the material's surface. The rendering of the measured ma-

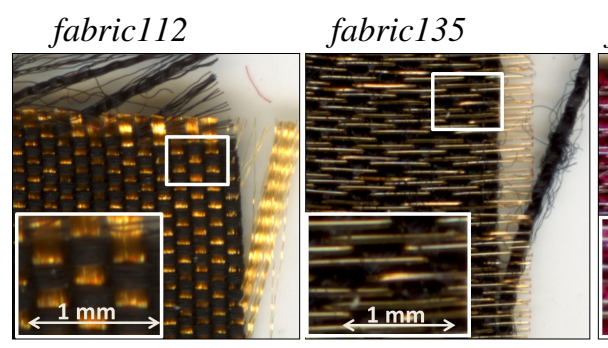

fabric136

Fig. 5. Detail structure of the measured materials.

terial's BRDF in environment illumination represented using 512 directional lights is shown in Fig. 2.

\section{Measured Data Evaluation}

We analyzed the quality of our datasets in several ways. We compared our data with: photographs of measured materials, alternative anisotropic BRDF measurements, sparse uniform sampling, and results of two state-of-the-art BRDF models.

Comparison with photographs - A comparison of measured BRDF with real appearance of measured fabrics was achieved by bending the fabrics along cylinder and photographed. Then the measured datasets were rendered on cylinder given the same conditions. Results shown in Fig. 6 illustrate good agreement between the measurements and their recorded BRDF. One has to take into account that the structure present in the photographs cannot by recorded by to BRDF.

Comparison with alternative measurements - We also compared our dense BRDF data with data provided by Ngan et al. [4]. These data collect 1924494 unique samples with valid RGB values for each of the four materials ${ }^{2}$; however, the

\footnotetext{
${ }^{1}$ http://www.cs.umd.edu/ mount/ANN/

${ }^{2} \mathrm{http}: / /$ people.csail.mit.edu/addy/research/brdf/
} 

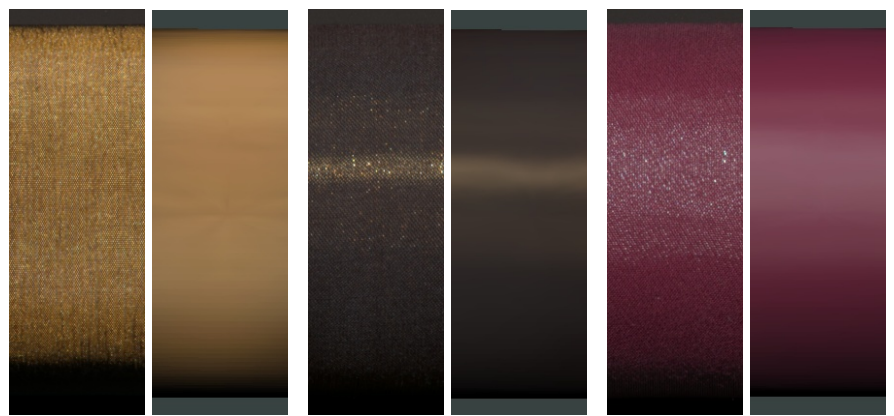

Fig. 6. Comparison between photos of the measured materials (left) and their BRDF renderings (right) on a cylinder illuminated from above the camera.

data are very noisy as shown on example of $\varphi_{i} / \varphi_{v}$ subspace interpolation for the fixed elevations $\theta_{i}=\theta_{v}=74^{\circ}$ in Fig. 7. Therefore, these datasets are better suited for fitting of BRDF models which can effectively suppress noise presented in the data, yet not suitable as a reference for example, for adaptive sampling.

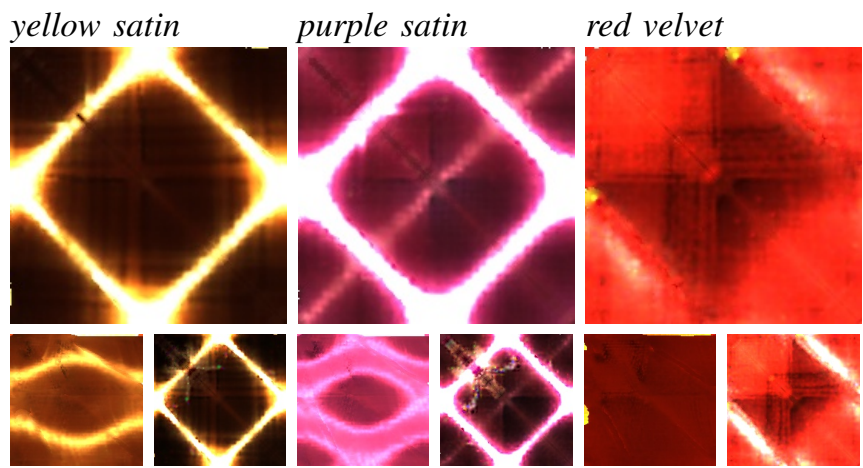

Fig. 7. BRDF subspace interpolated from BRDF data by Ngan et al. [4] (first row) at illumination/view elevations $74^{\circ} / 74^{\circ}$, (second row) for elevations $40^{\circ} / 16^{\circ}$ and $80^{\circ} / 80^{\circ}$.

For the sake of comparison, we selected two materials having similar properties to our first two materials of Ngan et al. (yellow satin and purple satin). One can observe the same BRDF subspace of our measurements in the first row of Fig. 8, where any visual artifacts are hardly noticeable. The last three columns of Fig. 8 also show rendering from the corresponding dataset for each measured material.

Comparison with sparse uniform sampling - One might suggest that sparse uniform sampling of BRDF space would perform similarly; therefore, we took only samples from the center of the measured images. In such a case, the hemisphere over the material was sampled by 81 illumination $\times 81$ view directions resulting in 6561 samples distributed uniformly over the hemisphere. When missing samples are interpolated using RBF up to density $2^{\circ}$, we can observe considerable differences, especially in improper reproduction of high frequency features e.g., width and continuity of specular highlights, as it is clearly visible in the second row of Fig. 8.

Fitting by BRDF models - For computational and data compression reasons BRDF data are typically represented by means of empirical or physically based models [5]. Although, these models represent directional-dependent material behavior by various analytical functions and thus efficiently filtering outliers in the data, they often tend to smooth and therefore simplify the data. Another challenge is the fitting of their parameters by optimization techniques that can be trapped in a local minimum. We tested two recent BRDF models. The first, a Kurt model [19] is an anisotropic extension of microfacet distribution-based Cook-Torance model. We fitted two lobes of this model and results are shown in the third row of Fig. 8. The second model [20] is based on physics of light scattering in cylindrical threads of fibers and achieves anisotropy by application of a user defined weaving pattern of two threads together with parameters of their reflectance model. A drawback of this model is a complicated application of parameter optimization techniques; therefore, derivation of appropriate parameters of individual threads is left completely to users and their experience. The results of our manual fitting of thread shapes and parameters are shown in the fourth row of Fig. 8. In our case, the last two materials contain tiny transparent nylon fibers instead of a cylindrical thread of fibers; difficult to reproduce reliably by the model.

Although application of both models yield significant errors for the observed subspace of rather extreme elevations, the renderings on sphere produce appearance similar to our measurements. These results underline the importance of accurate and dense BRDF measurements for BRDF related research as fitting of the analytical models always sacrifice a certain portion of apparent details.

Analysis of the measured datasets - As the size of the measured four-dimensional BRDF dataspace is enormous, we show only examples of all its possible slices in Fig. 9. Its first row shows nine subspaces of azimuthal angles $\varphi_{i} / \varphi_{v}$ for discrete elevation angles $\theta_{i} / \theta_{v}=\left[30^{\circ}, 60^{\circ}, 74^{\circ}\right]$. The materials fabric135 and fabric136 exhibit strong anisotropic highlights (bottom-left to top-right features) when illumination azimuth is perpendicular to nylon fibers reflecting light by their smooth surface (see Fig. 5). Specular highlights (topleft to bottom-right features) in near mirror reflections are of comparable intensity. The material fabricl12 exhibit a more complicated type of anisotropy as it consists of two threads of different properties (see Fig. 5). While both specular and single anisotropic highlights are comparable at low elevations (top-left subspace) with increasing elevation two types of anisotropic highlights appear: the yellow one for the gold thread and gray one for the dark thread. Due to mutual occlusion of both threads the intensity of specular highlights at high elevations depend on orientation of the material owing to camera and light and its color is determined by the prevailing dark thread. All materials show narrowing of specular features for high difference between illumination and view elevation angles $\left(\theta_{i}, \theta_{v}\right)$.

Fig. 9-b) shows 18 subspaces of angles $\theta_{i} / \varphi_{v}$ for discrete angles $\varphi_{i}=\left[0^{\circ}, 60^{\circ}, 120^{\circ}, 180^{\circ}, 240^{\circ}, 300\right] / \theta_{v}=$ $\left[30^{\circ}, 60^{\circ}, 74^{\circ}\right]$. These subspaces show distinct straight features whose orientation is dependent on $\varphi_{i}$ and offset on $\theta_{v}$. Fig. 9c) shows 18 subspaces of angles $\theta_{i} / \varphi_{i}$ for discrete angles $\varphi_{v}=\left[0^{\circ}, 60^{\circ}, 120^{\circ}, 180^{\circ}, 240^{\circ}, 300\right] / \theta_{v}=\left[30^{\circ}, 60^{\circ}, 74^{\circ}\right]$. These subspaces show distinct circular features whose diameter is dependent on $\theta_{v}$ and position is dependent on $\varphi_{v}$. Finally, Fig. 9-d) shows 36 subspaces of angles $\theta_{i} / \theta_{v}$ dependent on azimuthal angles $\varphi_{i} / \varphi_{v}$. A more detail analysis of the measured datasets is a subject of our future work. 


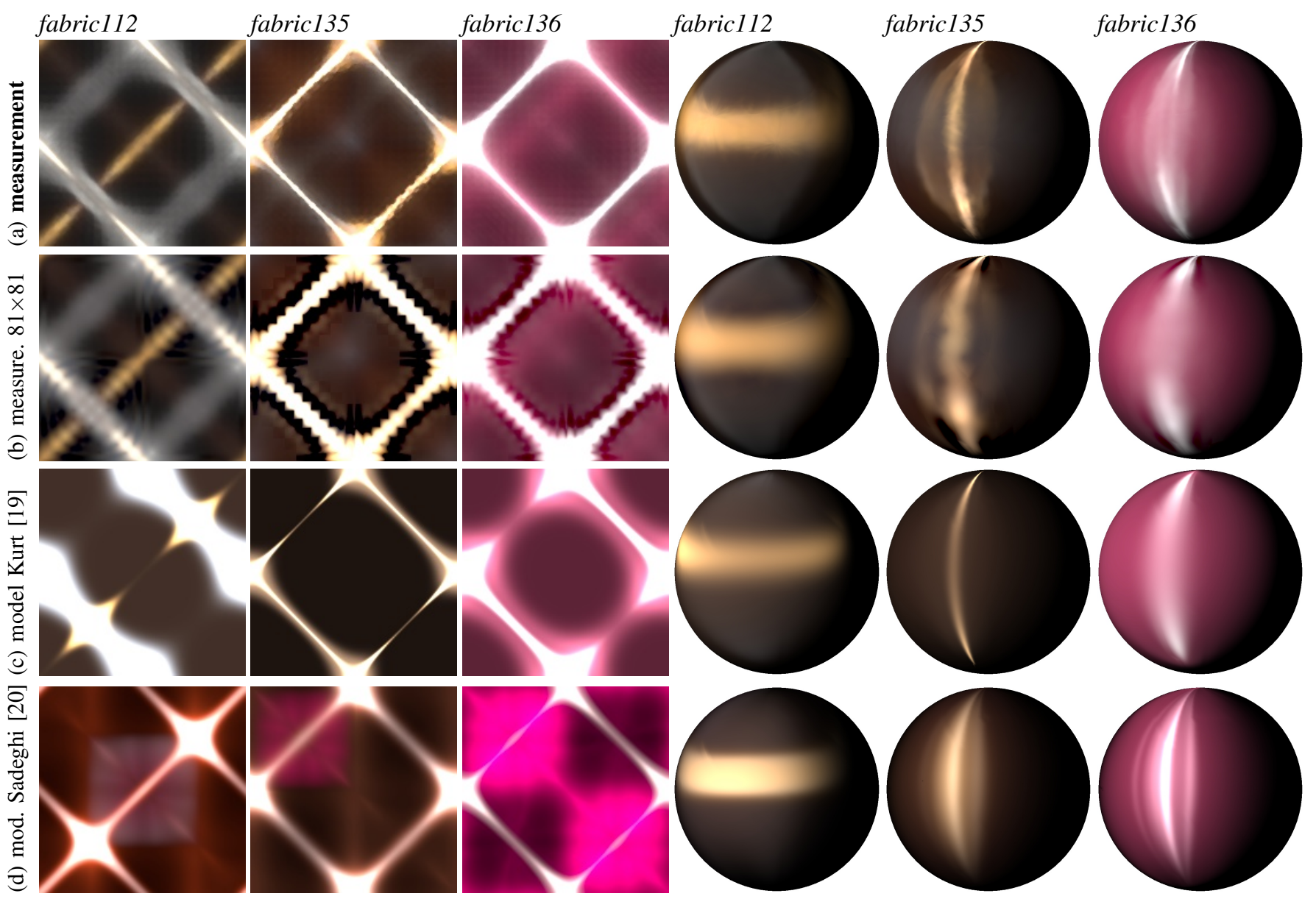

Fig. 8. BRDF subspace interpolated at illumination/view elevations $\theta_{i} / \theta_{v}=74^{\circ} / 74^{\circ}$ and BRDF renderings for the (a) measured dense data, (b) sparsely measured $(81 \times 81)$ data, and represented by (c) Kurt et al.[19] and (d) Sadeghi et al. [20] BRDF models.

\section{CONCLUSIONS}

An innovative image-based dense anisotropic BRDF measurement procedure using gonioreflectometer is introduced. We sample BRDF space uniformly while each sample represents an image of circular material probe of size comparable to distance from the material to light and camera. Due to the uniformity of material and slight variations of angles, we are able to collect many BRDF samples from each image. This approach allows for the acquisition of nearly three million samples from 8505 images. Therefore, our approach overcomes the main limitation of a gonioreflectometer based setup; the measurement time, as the measurement is completed withing twenty hours. This paper further deals with interpolation of the measured data into a dense grid. Finally, we compared the data with competing BRDF dataset and sparse uniform measurements as well as with results of two state-of-the-art physically-based BRDF models. We believe that due to the high accuracy of the data acquisition process, our publicly available measurements represent unique anisotropic data useful as a reference for further BRDF oriented research.

All the measured dense BRDF samples are available for research purposes as a part of the UTIA BTF Database ${ }^{3}$. Size of each BRDF in OpenEXR HDR format is $700 \mathrm{MB}$. Real material specimens can be provided upon request.

${ }^{3}$ http://btf.utia.cas.cz

\section{ACKNOWLEDGMENTS}

We would like to thank Oleg Bisker for help with implementation of the microcylinder BRDF model. This research has been supported by the Czech Science Foundation grants 103/11/0335, 14-02652S, and 14-10911S.

\section{REFERENCES}

[1] F. Nicodemus, J. Richmond, J. Hsia, I. Ginsburg, and T. Limperis, "Geometrical considerations and nomenclature for reflectance," NBS Monograph 160, National Bureau of Standards, U.S. Dept. of Com., pp. 1-52, 1977.

[2] K. Dana and J. Wang, "Device for convenient measurement of spatially varying bidirectional reflectance," Journal of Optical Society of America, vol. 21, no. 1, pp. 1-12, 2004.

[3] W. Matusik, H. Pfister, M. Brand, and L. McMillan, "A data-driven reflectance model," ACM Transactions on Graphics, vol. 22, no. 3, pp. 759-769, 2003.

[4] A. Ngan, F. Durand, and W. Matusik, "Experimental analysis of BRDF models," Eurographics Symposium on Rendering 2005, vol. 2, pp. 117126, 2005.

[5] M. Haindl and J. Filip, Visual Texture, ser. Advances in Computer Vision and Pattern Recognition. London: Springer-Verlag, 2013.

[6] J. Murray-Coleman and A. Smith, "The automated measurement of BRDFs and their application to luminaire modeling," Journal of the Illuminating Engineering Society, vol. 19, pp. 87-99, 1990.

[7] D. R. White, P. Saunders, S. J. Bonsey, J. van de Ven, and H. Edgar, "Reflectometer for measuring the bidirectional reflectance of rough surfaces," Journal of Applied Optics, vol. 37, pp. 3450-3454, June 1998.

[8] G. Ward, "Measuring and modeling anisotropic reflection," Computer Graphics, vol. 26, no. 2, pp. 265 - 272, July 1992. 


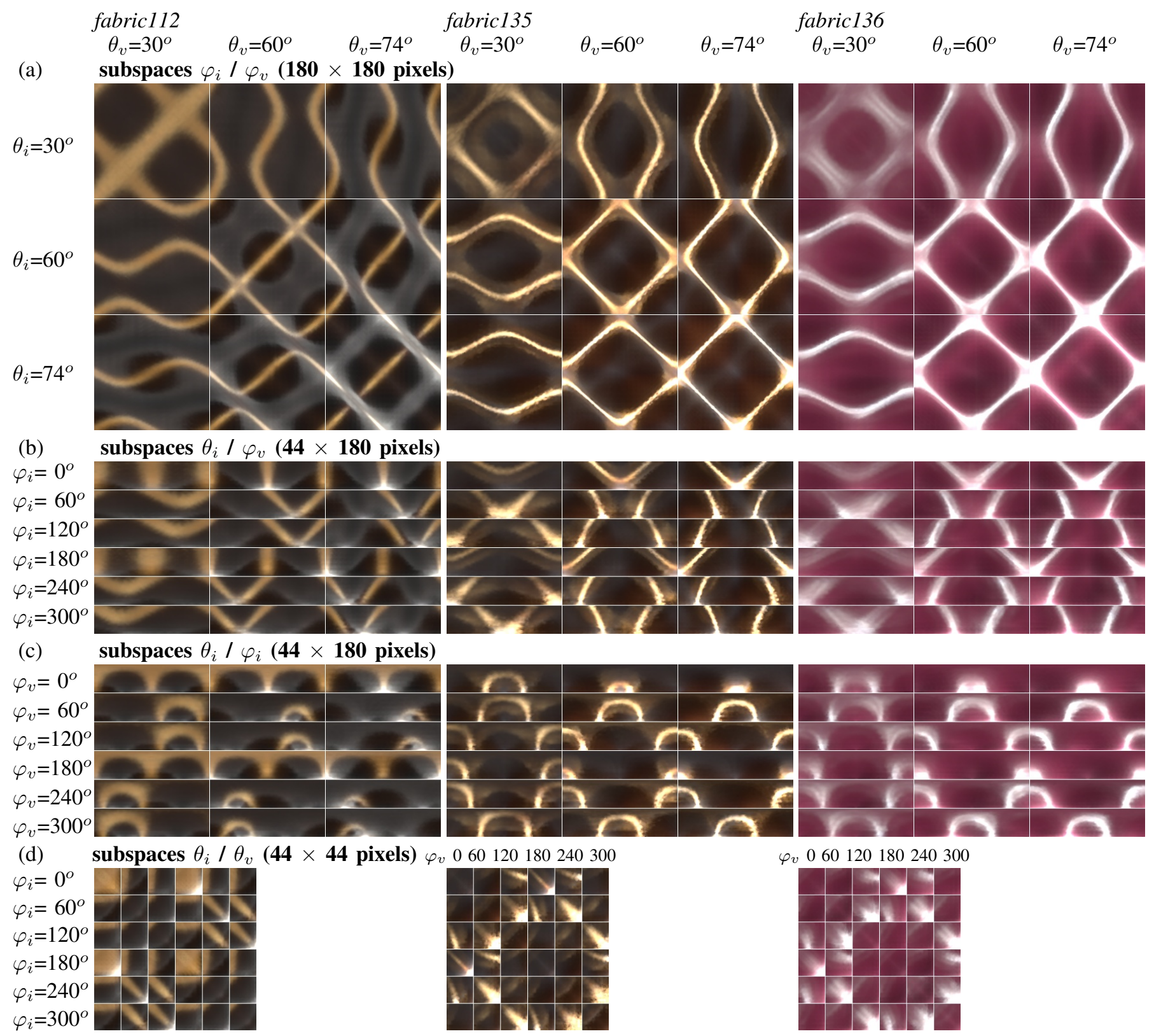

Fig. 9. Example of various BRDF 2D subspaces for the three measured materials: (a) illumination vs. view azimuth subspace $\left(\varphi_{i}, \varphi_{v}\right)$ for three different illumination/view elevation angles, (b) illumination elevation vs. view azimuth subspace $\left(\theta_{i}, \varphi_{v}\right)$, (c) illumination elevation vs. illumination azimuth subspace $\left(\theta_{i}, \varphi_{i}\right)$, and (d) illumination vs. view elevation subspace $\left(\theta_{i}, \theta_{v}\right)$.

[9] A. Ghosh, S. Achutha, W. Heidrich, and M. O'Toole, "BRDF acquisition with basis illumination," Computer Vision, IEEE International Conference on, vol. 0, pp. 1-8, 2007.

[10] K. Dana, "BRDF/BTF measurement device," in Proceedings of Eighth IEEE International Conference on Computer Vision. ICCV 2001, vol. 2, July 2001, pp. 460-466.

[11] M. Yasuhiro, K. Sumino, and Y. Yasushi, "Rapid BRDF measurement using an ellipsoidal mirror and a projector," IPSJ Trans. on Computer Vision and Appl., vol. 1, 21-32.

[12] W. Matusik, M. Pfister, H.P. Brand, and L. McMillan, "Image-based BRDF measurement including human skin," in Proceedings of 10th Eurographics Workshop on Rendering, 2003, pp. 139-152.

[13] S. R. Marschner, S. H. Westin, E. P. F. Lafortune, and K. E. Torrance, "Image-based bidirectional reflectance distribution function measurement," Applied Optics, vol. 39, 2000.

[14] R. Lu, J. J. Koenderink, and A. M. L. Kappers, "Optical properties (bidirectional reflectance distribution functions) of velvet." Applied Optics, vol. 37, pp. 5974-5984, 1998.
[15] M. Rump, G. Müller, R. Sarlette, D. Koch, and R. Klein, "Photo-realistic rendering of metallic car paint from image-based measurements," Computer Graphics Forum, vol. 27, no. 2, pp. 527-536, 2008.

[16] J. Filip, R. Vavra, M. Haindl, P. Zid, M. Krupicka, and V. Havran, "BRDF slices: Accurate adaptive anisotropic appearance acquisition," in CVPR 2013, 2013, pp. 4321-4326.

[17] M. Sattler, R. Sarlette, and R. Klein, "Efficient and realistic visualization of cloth," in Eurographics Symposium on Rendering, 2003, pp. 167-178.

[18] W. H. Press, S. A. Teukolsky, W. T. Vetterling, and B. P. Flannery, Numerical Recipes in C: The Art of Scientific Computing. Cambridge University Press, 1992.

[19] M. Kurt, L. Szirmay-Kalos, and J. Křivánek, "An anisotropic BRDF model for fitting and Monte Carlo rendering," SIGGRAPH Comput. Graph., vol. 44, pp. 3:1-3:15, February 2010.

[20] I. Sadeghi, O. Bisker, J. De Deken, and H. W. Jensen, "A practical microcylinder appearance model for cloth rendering," ACM Trans. Graph., vol. 32, no. 2, pp. 14:1-14:12, Apr. 2013. 